\title{
Gastric Mucosal Changes and Ghrelin Expression and Their Relation to Weight Reduction After Sleeve Gastrectomy
}

\author{
Sabry Ahmed Mahmoud ${ }^{1}$, Ahmed Fadaly Hussein ${ }^{1, ~ *, ~ W a l e e d ~ O m a r ~}{ }^{1}$, Emad Abdallah $^{1}$, \\ Wagdi Elkashef $^{2}$, Mohamed Anwar ${ }^{1}$, Sameh Emile ${ }^{1}$ \\ ${ }^{1}$ General Surgery Department, Mansoura University Hospitals, Mansoura University, Mansoura, Egypt \\ ${ }^{2}$ Pathology Department, Mansoura Faculty of Medicine, Mansoura University, Mansoura, Egypt
}

Email address:

Sabrybadr@hotmail.com (S. A. Mahmoud),dr.fadaly.1983@gmail.com(A. F. Hussein), waleed_h_omar@yahoo.com (W. Omar),

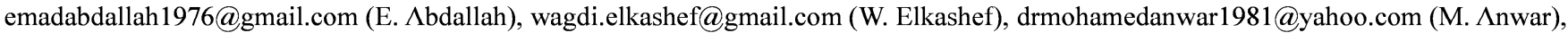
sameh200@hotmail.com (S. Emile)

${ }^{*}$ Corresponding author

\section{To cite this article:}

Sabry Ahmed Mahmoud, Ahmed Fadaly Hussein, Waleed Omar, Emad Abdallah, Wagdi Elkashef, Mohamed Anwar, Sameh Emile. Gastric Mucosal Changes and Ghrelin Expression and Their Relation to Weight Reduction After Sleeve Gastrectomy. Journal of Surgery.

Vol. 6, No. 2, 2018, pp. 36-42. doi: 10.11648/j.js.20180602.12

Received: February 6, 2018; Accepted: February 24, 2018; Published: March 19, 2018

\begin{abstract}
Background: Weight loss after laparoscopic sleeve gastrectomy (LSG) is usually attributed to the volume restrictive effect of the procedure in addition to specific hormonal changes. Objective: The present study aimed to investigate the changes in plasma ghrelin levels, the number of ghrelin producing cells and histopathological changes in the remaining pouch after LSG. Methods: The present study included 27 patients with morbid obesity. The plasma ghrelin levels were measured before and six months after LSG and the change in their levels was assessed in relation to body mass index (BMI) after LSG. Immunohistochemical staining of cellular ghrelin was used to evaluate the number and distribution of ghrelin producing cells in the resected specimen and the mucosal changes in the remaining gastric pouch after LSG were assessed at 6 months postoperatively. Results: The mean age of patients was $33.9 \pm 21.9$ years. At six months after LSG, BMI decreased from $52.6 \pm 12.8$ to $40.8 \pm 7.2 \mathrm{Kg} / \mathrm{m}^{2}$ ( $\mathrm{p}=0.0001$ ). The plasma ghrelin level decreased significantly from $564.1 \pm 15$ to $434.7 \pm$ 22.6 at six months after LSG. There was strong positive correlation between BMI after LSG and plasma ghrelin level $(\mathrm{R}=$ $0.906, p=<0.0001)$. A significant improvement in the inflammatory parameters was noticed by histopathologic examination. The mean ghrelin positive cell per specimen decreased significantly from $25.37 \pm 3.5$ to $13.7 \pm 2.12$. Conclusion: There was good positive correlation between weight loss and lowering of plasma ghrelin level, also complete removal of the fundus was associated with more weight loss.
\end{abstract}

Keywords: Sleeve Gastrectomy, Mucosal Changes, Ghrelin, Weight Loss

\section{Introduction}

Obesity is a pan-endemic health problem in both developed and developing countries that can be associated with a high incidence of complications and decrease in life expectancy, especially among younger adults. Obesity is classified into classes according to the body mass index (BMI) with morbid obesity defined as BMI $\geq 40 \mathrm{~kg} / \mathrm{m}^{2}$ or a BMI $\geq 35 \mathrm{~kg} / \mathrm{m}^{2}$ in patients with at least one associated comorbidity [1].
While conservative measures may succeed in treatment of obesity in some patients, bariatric surgery remains a viable option for the treatment of morbid obesity, resulting longlasting weight loss, improved quality of life, and resolution of obesity- related comorbidities [2]. With the application of laparoscopy to bariatric surgery a major expansion in the number of laparoscopic bariatric procedures has been noted worldwide [3].

Laparoscopic sleeve gastrectomy (LSG) is one of the most popular bariatric procedures performed for morbid obesity 
[4]. The mechanism of weight loss after sleeve gastrectomy is attributed to a combination of volume restriction, creation of a high pressure system, and the induction of a favorable hormonal change [5].

A decline in appetite after LSG has been observed, presumably due, in part, to ghrelin cell removal. Ghrelin is an orexigenic hormone produced primarily by cells in the oxyntic glands of the stomach mainly in the gastric fundus as it has been demonstrated that the expression of Ghrelin mRNA expression in the fundus is three fold higher than in the pre-pyloric area and the same pattern was also seen in ghrelin cell distribution [6].

Ghrelin hormone plays a physiologic role in the regulation of food intake as its plasma levels rise during food deprivation and decline after food ingestion [7]. In addition, Ghrelin is involved in many biological processes ranging from appetite regulation and growth hormone release to gut motility [8]. The changes in plasma ghrelin levels after different bariatric procedures vary according to the procedure. While plasma ghrelin levels tend to decrease after gastric bypass and after LSG, they tend to rise after gastric banding in a way similar to dieting [9].

The present study aimed to evaluate the changes in plasma ghrelin hormone at six months after LSG, correlating these changes with weight loss. The study also aimed to investigate the distribution of ghrelin producing cells in different regions of the stomach and the change in their number after LSG and to evaluate the histopathological changes after sleeve gastrectomy since the evaluation of these changes can improve the understanding of the local mechanism and the outcome of LSG as Vrabie et al. implied [10].

\section{Patients and Methods}

This is a prospective cohort study on patients with morbid obesity who underwent LSG in the General Surgery Department of Mansoura University Hospitals in the period of May 2015 to May 2017. Ethical approval of the study was obtained from the Institutional Review Board (IRB) of Mansoura Faculty of Medicine.

All patients with morbid obesity with BMI $>40 \mathrm{~kg} / \mathrm{m}^{2}$ or BMI $>35 \mathrm{~kg} / \mathrm{m}^{2}$ with at least one obesity-related comorbidity who failed conservative treatments were included to the study.

We excluded patients who underwent previous bariatric surgery, patients unfit for anesthesia as patients, patients with gastric pathologies as peptic ulcer or neoplasm, patients with secondary obesity due to endocrine disorders, patients with psychological problems or patients unwilling to comply with postoperative diet regimen.

Patients were asked about the onset and duration of obesity and any obesity-related comorbidities such as diabetes mellitus, hypertension, sleep apnea, and joint pain. Patients were also asked about previous treatments for morbid obesity including diet regimens, weight loss medications, exercise programs, interventional therapies as intragastric ballon, and any previous surgical procedures for morbid obesity or other abdominal conditions. Detailed dietary history was taken from all patients with regards to type and frequency of meals, eating snacks in between meals, drinking of water or beverages, level of appetite, and feeling of satiety after each meal.

General examination was conducted to all patients. Patients' weight and height were recorded and BMI was then calculated using the following equation: $\mathrm{BMI}=$ Weight in kilograms/ (Height in meters) ${ }^{2}$. Abdominal examination was performed to exclude presence of any abdominal masses, ascites, and ventral or groin hernias.

Routine preoperative laboratory investigations including complete blood count, liver and kidney functions tests, prothrombin time, and random blood glucose were done to confirm anesthetic fitness. Endocrine and metabolic panels including serum lipid profile, thyroid function tests, and fasting and postprandial blood sugar levels were done to exclude secondary causes of obesity. Plasma ghrelin levels were measured in all patients within 48 hours before the procedure.

Abdominal ultrasonography was performed in all patients to exclude any associated abdominal pathologies. The cardiac function was evaluated by electrocardiography and echocardiography in select patients and pulmonary function tests were done to assess respiratory functions and chest condition.

Informed consent for the study was taken from all patients. Liquid diet was prescribed for 24 hours before the procedure with fasting for at least six hours before the onset of anesthesia.

Preoperative measures against thromboembolism were taken in the form of: wearing elastic stocking 24 hours before the operation, subcutaneous administration of low molecular weight heparin (Enoxaprin, $40 \mathrm{IU}$ ) at the night of surgery. Prophylactic antibiotics (cefotaxime $1 \mathrm{gm}$ ) were administered intravenously on induction of anesthesia.

All procedures were done under general anesthesia with end tracheal intubation. Patients were placed in the supine position being appropriately secured to the operating table with padding of all pressure points. The operating surgeon stood between the legs of the patient and the two assistants stood on each side of the patient.

After scrubbing of the abdomen with povidone iodine, peumoperitoneum $(15-17 \mathrm{mmHg})$ was established by insertion of veress needle above the umbilicus and insufflation of $\mathrm{CO} 2$. Trocars were inserted under direct vision as follows: a 5-mm trocar is inserted in the subxiphoid area for the liver retractor, another 5-mm trocar is placed in the left upper quadrant at the anterior axillary line just below the $12^{\text {th }}$ rib for the assistant, additional $12-\mathrm{mm}$ trocars were placed in the right upper quadrant, epigastrium, left upper quadrant, and right paramedian regions for inserting staplers.

Upon identification of the pylorus by visualizing the prepyloric vein of Mayo, the greater curvature of the stomach was devascularized using an advanced vessel-sealing device (Harmonic scalpel $^{\mathrm{TM}}$ or Ligasure ${ }^{\mathrm{TM}}$ ) starting at $6 \mathrm{~cm}$ away from the pylorus. Devascularization continued proximally 
onto the fundus until the left crus of the diaphragmatic hiatus was clearly identified. All the short gastric vessels were divided along the way. After devascularizaion was completed, the hiatus was assessed for hernias and if large hiatal hernia was encountered, it was repaired using standard laparoscopic techniques.

A 36 French bougie was then inserted transorally by the anesthesiologist, advanced under direct vision to the pylorus, and then positioned against the lesser curvature. The gastric transection was then performed using sequential applications of $60-\mathrm{mm}$ linear staplers beginning at $6 \mathrm{~cm}$ proximal to the pylorus. As the gastric transection proceeds, the height of the staples may need to be adjusted according to the thickness of the tissue. We used green stapler at pylorus, then gold staplers at middle of stomach, then blue staplers at fundus.

After the stomach was transected, an intraoperative leak test was performed by injection of methylene blue in the gastric sleeve. The resected portion of the stomach was extracted through the right upper quadrant trocar then the trocar sites were closed and an intra-abdominal drain was inserted.

Patients were monitored in the regular ward with regards vital signs, output of abdominal drain, and postoperative complications. Intravenous fluids were administered for 24 hours postoperatively and one gram of cefotaxime was administered intravenously every 12 hours. Analgesia was achieved using non-steroidal anti-inflammatory drugs (NSAIDs) or Nalufin amp. A second dose of low molecular weight heparin was given subcutaneously 8 hours after the operation then was repeated every 24 hours. Early ambulation was advised in all patients. Resumption of oral feeding was scheduled in every patient. Once assured to be stable, started oral fluids, with no complaints, patients were discharged home within 48 hours of the procedure.

The sleeve gastrectomy specimens were fixed in $4 \%$ formaldehyde and embedded in paraffin, and $4 \mathrm{~mm}$ thick sections from each specimen were stained with hematoxylin and eosin for histopathological examination. The biopsy specimens were examined in a blind manner by a pathologist to indicate:

1- Presence of lymphoid follicles (Scored 0: absent, 1: mild, 2: moderate, 3: Marked). 2- Interstitial inflammation (Scored 0: absent, 1: mild, 2: moderate, 3: Marked).

Ghrelin immunoreactivity was determined on sections after microwave pretreatment $(10 \mathrm{~min}$ in $0.01 \mathrm{M}$ citric acid solution) and overnight incubation at $4{ }^{\circ} \mathrm{C}$ with human polyclonal antibody (Chongqing biopsies Co., Ltd., Chongqing, China) diluted 1:100. Diaminobenzidinehydrogen peroxide was used as chromogen, and the sections were counterstained with diluted hematoxylin.

Ghrelin positive cells were counted in three (hot spots) in areas containing mucosa in full thickness. The total number was divided by 3 to obtain mean Ghrelin positive cell per specimen.

Patients were asked to visit the outpatient clinic at one and two weeks after sleeve gastrectomy for assessment of general condition, wound status, and for removal of stitches, then every month for six months.

At Six months after the procedure, body weight was recorded and the amount and percentage of weight loss was calculated and compared with the preoperative value. BMI was calculated to estimate the reduction in BMI after surgery. A blood sample was withdrawn and plasma ghrelin levels were measured and compared to the preoperative value. In addition, all patients underwent gastroscopy to evaluate the condition of mucosa of the remaining pouch and take three tissue samples from the remaining part of the stomach.

Tissue samples were examined pathologically with the same techniques described earlier to assess postoperative changes in ghrelin gene expression, mucosal cells distribution and to demonstrate any histopathological changes.

Data were analyzed using SPSS program version 23 (IBM corp., Bristol, UK). Continuous variables were expressed as mean and standard deviation (SD) or median and normal range. Categorical variables were expressed as number and percentages. Student t-test was used for processing of quantitative data whereas Fisher's exact test was used for processing of qualitative data. The correlation between BMI and plasma ghrelin levels was measured using Pearson correlation test. $\mathrm{P}$ value less than 0.05 was considered significant.

\section{Results}

The present study included 27 patients with morbid obesity who underwent LSG. Patients were all females except one male. The mean age of patients was $33.9 \pm 21.9$ years, ranging from 21 to 63 years.

Twenty-four patients had obesity-related comorbidities: nine patients had type II DM, five had arterial hypertension, ten had dyslipidemia, and four had joint pain. In addition, 12 patients had fatty liver and two had gall bladder stones in abdominal ultrasonography.

The mean preoperative body weight was $137 \pm 3.5$ (range, 108- 164) Kg. The mean preoperative height of patients was $162.4 \pm 21.2$ (range, 148- 183) $\mathrm{cm}$. The mean preoperative BMI was $52.6 \pm 12.8$ (range, 38.8- 66.6) $\mathrm{Kg} / \mathrm{m}^{2}$.

The mean operating time was $104.2 \pm 14.1$ (range, 80-135) minutes. The mean hospital stay was $2.3 \pm 0.4$ (range, 2-4) days. There was no conversion to open surgery in any patient. Intraperitoneal drains were placed in $21(77.7 \%)$ patients.

No major complications or mortality were recorded. Minor complications were recorded in 12 patients, wound infection in 3 patients, chest infection in 5 patients, and vomiting in 4 patients. Simultaneous cholecystectomy was conducted with LSG in two patients.

At six months postoperatively, significant weight loss was noted as the mean preoperative body weight dropped from $137 \pm 3.5$ to $107 \pm 2.12 \mathrm{Kg}(\mathrm{p}<0.0001)$. The preoperative BMI decreased from $52.6 \pm 12.8$ to $40.8 \pm 7.2 \mathrm{Kg} / \mathrm{m}^{2}$ ( $\mathrm{p}=$ $0.0001)$. The mean percentage of excess weight loss was $41.7 \pm 11.1$ (range, 29.3- 64.4) (table 1). 
Table 1. Weight loss after sleeve gastrectomy.

\begin{tabular}{llll}
\hline Variable & Preoperative & Postoperative & P value \\
\hline Body weight & $137 \pm 3.5$ & $107 \pm 2.12$ & $<0.0001$ \\
BMI & $52.6 \pm 12.8$ & $40.8 \pm 7.2$ & $<0.0001$ \\
$\%$ EWL & ------ & $41.7 \pm 11.1$ & ----- \\
\hline
\end{tabular}

Improvement in obesity associated comorbidities was observed in $6(66.7 \%)$ of nine diabetic patients, three $(60 \%)$ of five patients with hypertension, and one $(25 \%)$ of four patients with joint pain (table 2).

Table 2. Improvement in co-morbidities loss after sleeve gastrectomy.

\begin{tabular}{llll}
\hline Variable & Preoperative (\%) & Postoperative (\%) & P value \\
\hline D. M & $9(33.3)$ & $3(11.1)$ & 0.1 \\
Hypertension & $5(18.5)$ & $2(7.4)$ & 0.42 \\
Joint pain & $4(14.8)$ & $3(11.1)$ & 1 \\
\hline
\end{tabular}

The plasma ghrelin level decreased significantly from $564.1 \pm 15$ to $434.7 \pm 22.6$ at six months after LSG $(\mathrm{p}<$ $0.0001)$. There was weak positive correlation between preoperative BMI and plasma ghrelin level $(\mathrm{R}=0.089$, $\mathrm{p}=$ 0.65). On the other hand, there was strong positive correlation between BMI after LSG and plasma ghrelin level $(\mathrm{R}=0.906, \mathrm{p}=<0.0001)$. A moderate negative correlation between $\% \mathrm{EWL}$ and postoperative plasma ghrelin level was observed $(\mathrm{R}=-0.652, \mathrm{p}=0.0002)$ (figure 1).

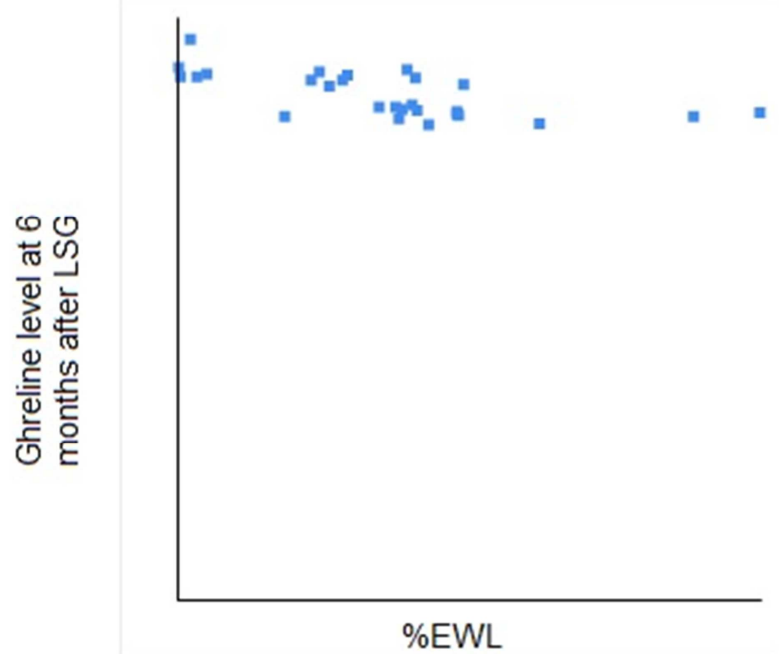

Figure 1. Correlation between excess weight loss and ghrelin level at 6 months after $L S G$.

Histopathological examination of the postoperative specimen and random endoscopic biopsies from the remaining gastric pouch revealed the following (table 3):

1. Superficial gastritis was detected in six patients (4 moderate and 2 mild) and superficial congestion in two patients.
2. Regarding the presence of lymphoid follicles, the number of patients with grade 0 increased from 15 to 22 , the number of patients with grade I decreased from 9 to 5 , and the number of patients with grade II decreased from 3 to 0 $(p=0.059)$ (figure 2).

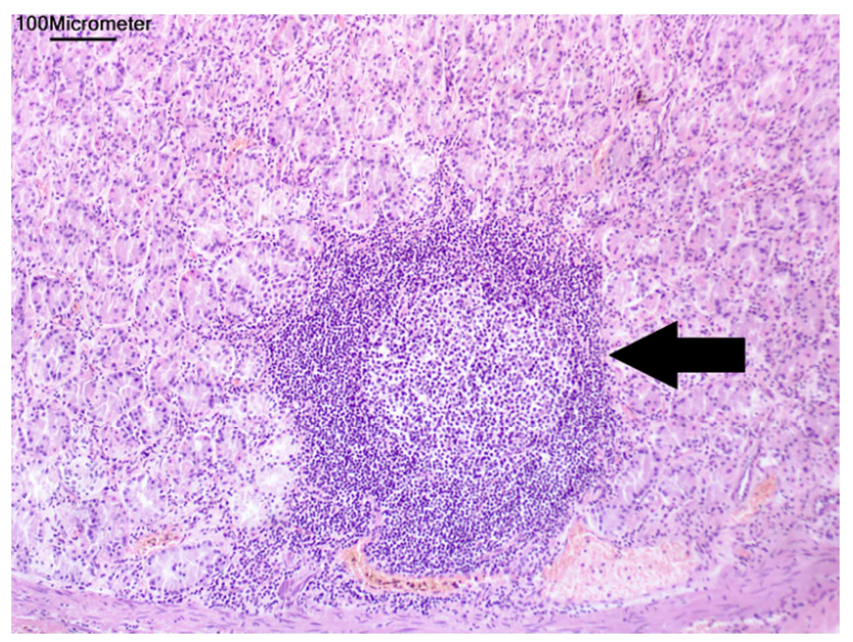

Figure 2. Gastric mucosa with lymphoid follicle (arrow) (H\&E stain, 100x).

3. Regarding the presence of interstitial inflammation, the number of patients with grade 0 increased from 12 to 20 , the number of patients with grade I decreased from 12 to 7 , and the number of patients with grade II decreased from 3 to 0 $(\mathrm{p}=0.03)$ (figure 3$)$.

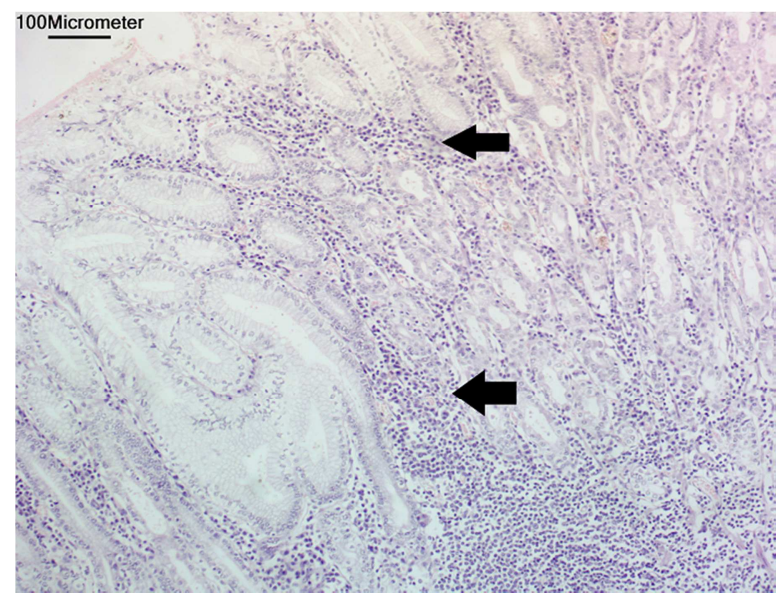

Figure 3. Gastric mucosa with interstitial inflammatory reaction (arrows) (H\&E stain, 100x).

4. The mean ghrelin positive cell per specimen decreased from $25.37 \pm 3.5$ to $13.7 \pm 2.12(\mathrm{p}<0.0001)$.

5 . The mean number of ghrelin producing cells in the fundus of the resected specimen was significantly higher than the body and the antrum as shown in table 4 and figure 4.

Table 3. Histopathological changes in the surgical specimen and remaining pouch after sleeve gastrectomy.

\begin{tabular}{llll}
\hline Variable & & Surgical specimen & Remaining gastric pouch \\
\hline \multirow{3}{*}{ Lymphoid follicle } & Grade 0 & $15(55.5)$ & $22(81.4)$ \\
& Grade 1 & $9(33.3)$ & $5(18.5)$ \\
Interstitial inflammation & Grade 2 & $3(11.1)$ & 0 \\
\hline
\end{tabular}




\begin{tabular}{llll}
\hline Variable & & Surgical specimen & Remaining gastric pouch \\
\hline & Grade 1 & $12(44.4)$ & $7(25.9)$ \\
Grade 2 & $3(11.1)$ & 0 \\
\hline
\end{tabular}

Table 4. Immunohistochemical distribution of ghrelin producing cells in the regions of the resected specimen.

\begin{tabular}{|c|c|c|c|c|c|}
\hline Variable & The fundus & The body & P value & The antrum & $P$ value \\
\hline Ghrelin producing cells (mean \pm SD) & $15 \pm 3.7$ & $7.1 \pm 3.1$ & $<0.0001$ & $3.3 \pm 1.8$ & $<0.0001$ \\
\hline
\end{tabular}

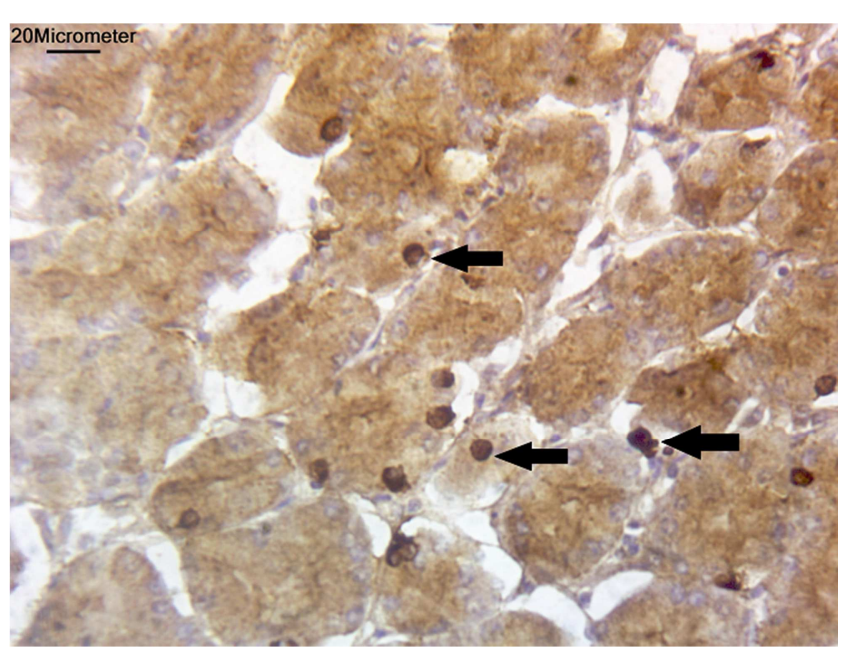

Figure 4. Gastric mucosa with positive reaction to Ghrelin immunohistochemistry (Brown staining, arrows) (Ghrelin immunohistochemical staining, 400x).

\section{Discussion}

Twenty-seven patients with morbid obesity were admitted and underwent LSG in this prospective observational study. Almost all patients were females reflecting the female predominance of obesity as documented in the literature. The majority of patients were of middle age in concordance with the average age of patients undergoing bariatric surgery for morbid obesity [4].

Significant weight loss at 6 months after LSG was recorded with a significant decline in the preoperative BMI. The average percentage of excess weight loss was around $40 \%$. Improvement in associated co-morbidities was observed yet was not statistically significant, perhaps due to the short duration of follow-up.

We studied plasma ghrelin levels before and after LSG, correlating the changes in these levels with weight loss after the procedure. A significant decrease in plasma ghrelin levels at 6 months after LSG was recorded which was associated with significant weight loss as demonstrated by decline in BMI. In concordance, other researchers found total plasma ghrelin levels decrease significantly at 3 months after LSG, with simultaneous significant weight loss [11].

Plasma ghrelin levels have been shown to decrease after sleeve gastrectomy and its low levels can be maintained up to five years after surgery $[12,13]$. Since ghrelin is mainly produced by oxyntic cells in the stomach in addition to other parts of the gastrointestinal tract, it is logical that plasma ghrelin levels decline after resecting around $75 \%$ of the stomach volume in LSG [14].
We identified a weak correlation between plasma ghrelin levels and BMI before surgery, nevertheless this positive correlation became stronger at 6 months after LSG and this correlation was statistically significant. Similarly, the percentage of excess weight loss at 6 months had moderately strong negative correlation with plasma ghrelin implying that the drop in plasma ghrelin levels was associated with higher percentage of weight loss. In discordance, Goitein et al. [11] found no distinct correlation between plasma ghrelin levels and weight loss which they explained that plasma ghrelin is only one of multiple factors contributing to the success of LSG and that weight loss per se is not responsible for ghrelin level change [15].

The significant strong correlation we observed between plasma ghrelin and BMI after surgery can be attributed to different patient characteristics compared to the study of Goitein et al. [11] where the BMI of our patients was obviously higher than their study. In addition, we made the correlation at six months after LSG, whereas the other investigators made the same correlation at shorter period of follow-up (three months postoperatively).

Ghrelin hormone has been recognized to contribute to the incidence of morbid obesity through different mechanisms. Ghrelin is one of the orexigenic peptide transmitters know to stimulate appetite and induce a positive energy balance leading to weight gain. It is implicated in regulating mealtime hunger and meal initiation. Since ghrelin hormone is considered a signal of starvation, it can induce sustained adiposity as long as its levels are elevated [16].

Histopathological examination of the resected part of the stomach after LSG revealed abnormal pathologic changes in $55.5 \%$ of patients in the present study. This implies that around $45 \%$ of patients had normal histopathology of the stomach preoperatively which is lower than other studies [1719] that reported normal histopathology in 50-54\% of patients. However, similar to our findings other investigators reported normal histopathology of the stomach in 35.2 and $46.3 \%$ of patients $[20,21]$.

Abnormal histopathological findings in the present study involved chronic gastritis, either active or inactive which is in line with Safaan et al. [17] who reported chronic gastritis to be the most common abnormal histopathological findings in the resected part of the stomach after LSG. Nonetheless, the study by Safaan and colleagues [17] documented other important pathological findings as intestinal metaplasia, GIST, leiomyoma, lipoma, and pancreatic heterotropia, however, these abnormal findings were found in a very low incidence of patients. In fact, the incidence of GIST ranged between 0.2 and $1 \%$ and the incidence of intestinal 
metaplasia ranged between 0.2 and $1.4 \%$ across several studies [17-23].

No incidental gastric malignancy in the postoperative specimen was detected in our study, in concordance with largest study [17] of histopathological diagnoses in LSG patients. The observation of lack of malignancies further raises the question of any added value of routine histopathological examination of the resected part of the stomach after LSG as AbdullGaffar et al. [18] implied. In light of financial restrictions that warrants evidence-based decision-making based on the cost-effectiveness concept, routine histopathological examination of surgical specimens has been previously questioned [24, 25].

We used two markers to measure inflammatory activity in gastric specimen after LSG and in the remaining pouch on follow-up. Presence of lymphoid follicles and interstitial inflammation were graded from 0 to 3 according to their severity. Presence of lymphoid follicles was observed in around $45 \%$ of patients in the resected specimen and in less than $20 \%$ of patients in the remaining gastric pouch in agreement with Onzi et al. [26]. who also reported decrease in inflammatory activity from $33 \%$ to less than $10 \%$ of patients in the remaining pouch after LSG

Similarly, $55.5 \%$ of patients showed evidence of interstitial inflammation of varying degree in the postoperative specimen which decreased significantly to around $25 \%$ in the remaining pouch at 6 months of follow-up. The improvement of the inflammatory parameters may be due to less exposure of patients to aggressive dietary factors owing to the restrictive effect of LSG as Onzi and coworkers [26] stated.

Additionally, a relation between chronic gastritis and Helicobacter pylori infection has been described in approximately $75 \%$ of asymptomatic patients, indicating that these bacteria could be an initiating factor of the process of gastritis [27]. Onzi et al. [26] verified the relation between Helicobacter pylori and chronic gastritis in obese patients because all patients with chronic gastritis in their study also had Helicobacter pylori according to positive urease test. Since we did not examine for the presence of Helicobacter pylori infection in the patients studied, we cannot conclude similar findings.

The number of ghrelin positive cells showed a significant decrease in the remaining pouch after LSG. This marked decrease can be explained that ghrelin positive cells are mainly located in the gastric fundus, which is removed in LSG, and tend to decrease in number towards the antrum. This decrease of ghrelin cell count along the stomach is consistent with similar findings demonstrating higher levels of expression in endoscopically obtained samples from the fundus and antrum of healthy individuals [28].

Regarding the prevalence of ghrelin-secreting cells detected by immunohistochemical staining, the number of ghrelin positive cells in the gastric fundus was significantly higher than the antrum. In contrast, Goitein et al. [11] found the difference in ghrelin cell count between fundus, body, and antrum of the stomach to be statistically insignificant, perhaps due to the small sample size included in their study.
The presence of ghrelin-secreting cells in the antrum, though small in number, is of special interest. The removal of antrum was correlated with sustained weight loss on the long term, perhaps due to removal of additional ghrelin-producing cells in the antrum. Leaving intact antrum may lead to weight regain partly due to preservation of ghrelin producing cells in the antrum and partly due to sleeve dilatation which minimizes the restrictive effect of LSG. On the other hand, preservation of the pumping mechanism of the antrum may serve to facilitate gastric empting [29, 30].

The significant decrease in ghrelin producing cells after LSG was paralleled by a similar decrease in plasma ghrelin level and significant weight loss at 6 months postoperatively. Weight regain in some patients after LSG was linked to the formation of neo-fundus as demonstrated in follow-up contrast studies [31]. Neo-fundus is formed by stretching and enlargement of the upper part of the remaining gastric sleeve and is associated with slow increase in plasma ghrelin level, though it remains lower than the preoperative levels [32].

Limitations of the current study include the small number of patients included and short follow-up. Furthermore, the presence of Helicobacter pylori in the resected specimen and the remaining gastric pouch was not investigated which may have a relation with the improvement in inflammatory activity postoperatively.

\section{Conclusion}

There was good positive correlation between weight loss and lowering of plasma ghrelin level, also complete removal of the fundus was associated with more weight loss. Similarly, a significant decrease in the number of ghrelin-producing cells was noted after LSG with evidence of higher number of ghrelin positive cells in the gastric fundus compared to the body and pre-antral regions. Improvement in inflammatory parameters as demonstrated by resolution of lymphoid follicles and interstitial inflammation was also observed.

\section{Conflict of Interest}

None to be disclosed by the authors

\section{References}

[1] Fontaine KR, Redden DT, Wang C, et al. (2003): Years of life lost due to obesity. JAMA; 289:187-193.

[2] Buchwald H, Avidor Y, Braunwald E, et al. (2004): Bariatric surgery: asystematic review and meta-analysis. JAMA; 292:1724-37.

[3] Cottam D, Mattar S, Schauer P (2003): Laparoscopic era of operations for morbid obesity. Arch Surg; 138: 367-75.

[4] Emile SH, Elfeki H, Elalfy K, Abdallah E. Laparoscopic Sleeve Gastrectomy Then and Now: An Updated Systematic Review of the Progress and Short-term Outcomes Over the Last 5 Years. Surg Laparosc Endosc Percutan Tech. 2017 Oct; 27 (5):307-317. doi: 10.1097/SLE.0000000000000418. 
[5] de Zwaan M, Mitchell JE, Howell LM et al. Characteristics of morbidly obese patients before gastric bypass surgery. Compr Psychiatry 2003; 44:428-34.

[6] Tschop M, Wawarta R, Riepl RL, et al. Post-prandial decrease of circulating human ghrelin levels. J Endocrinol Invest. 2001; 24: RC19-21.

[7] Shiiya T, Nakazato M, Mizuta M, et al. Plasma ghrelin levels in lean and obese humans and the effect of glucose on ghrelin secretion. J Clin Endocrinol Metab. 2002; 87:240-4.

[8] Date Y, Kojima M, Hosoda H, et al. Ghrelin, a novel growth hormone-releasing acylated peptide, is synthesized in a distinct endocrine cell type in the gastrointestinal tracts of rats and humans. Endocrinology. 2000; 141:4255-61.

[9] Cummings DE, Weigle DS, Frayo RS, et al. Plasma ghrelin levels after diet-induced weight loss or gastric bypass surgery. N Engl J Med. 2002; 346:1623-30.

[10] Vrabie C, Cojocaru M, Waller M, Sindelaru R, Copaescu C. The main histopathological gastric lesions in obese patients who underwent sleeve gastrectomy. Dicle Med J Cilt / Vol 37, No 2, 97-103.

[11] Goitein D, Lederfein D, Tzioni R, Berkenstadt H, Venturero M, Rubin M. Mapping of Ghrelin Gene Expression and Cell Distribution in the Stomach of Morbidly Obese Patients-a Possible Guide for Efficient Sleeve Gastrectomy Construction. OBES SURG (2012) 22:617-622. DOI 10.1007/s11695-0110585-9.

[12] Bohdjalian A, Langer FB, Shakeri-Leidenmuhler S, et al. Sleeve gastrectomy as sole and definitive bariatric procedure: 5-year results for weight loss and ghrelin. Obes Surg. 2010; 20:535-40.

[13] Langer FB, Reza Hoda MA, Bohdjalian A, et al. Sleeve gastrectomy and gastric banding: effects on plasma ghrelin levels. Obes Surg. 2005; 15:1024-9.

[14] Ariyasu H, Takaya K, Tagami T, et al. Stomach is a major source of circulating ghrelin, and feeding state determines plasma ghrelin-like immunoreactivity levels in humans. J Clin Endocrinol Metab. 2001; 86:4753-8.

[15] Yehoshua RT, Eidelman LA, Stein M, et al. Laparoscopic sleeve gastrectomy-volume and pressure assessment. Obes Surg. 2008; 18:1083-8.

[16] le Roux CW1, Neary NM, Halsey TJ, Small CJ, Martinez-Isla AM, Ghatei MA, et al. Ghrelin does not stimulate food intake in patients with surgical procedures involving vagotomy. J Clin Endocrinol Metab. 2005 Aug; 90 (8):4521-4. Epub 2005 May 24.

[17] Safaan $T$ \& Bashah $M$ \& El Ansari W \& Karam M. Histopathological Changes in Laparoscopic Sleeve Gastrectomy Specimens: Prevalence, Risk Factors, and Value of Routine Histopathologic Examination. OBES SURG (2017) 27:1741-1749.

[18] AbdullGaffar B, Raman L, Khamas A, et al. Should we abandon routine microscopic examination in bariatric sleeve gastrectomy specimens? Obes Surg. 2016; 26 (1):105-10.
[19] Clapp B. Histopathologic findings in the resected specimen of a sleeve gastrectomy. JSLS. 2015; 19 (1):e2013.00259. doi:10.4293 /JSLS.2013.00259.

[20] Raess PW, Baird-Howell M, Aggarwal R, et al. Vertical sleeve gastrectomy specimens have a high prevalence of unexpected histopathologic findings requiring additional clinical management. Surg Obes Relat Dis. 2015; 11 (5):1020-3.

[21] Lauti M, Gormack SE, Thomas JM, et al. What does the excised stomach from sleeve gastrectomy tell us? Obes Surg. 2016; 26 (4): 839-42.

[22] Almazeedi S, Al-Sabah S, Al-Mulla A, et al. Gastric histopathologies in patients undergoing laparoscopic sleeve gastrectomies. Obes Surg. 2013; 23:314-9.

[23] Ohanessian SE, Rogers AM, Karamchandan DM. Spectrum of gastric histopathologies in severely obese American patients undergoing sleeve gastrectomy. Obes Surg. 2016; 26 (3):595602 .

[24] Lohsiriwat VL, Vongjirad A, Lohsiriwat D. Value of routine histopathologic examination of three common surgical specimens: appendix, gallbladder, and hemorrhoid. World J Surg. 2009; 33 (10): 2189-93.

[25] van Vliet JL, van Gulik TM, Verbeek PC. Is it necessary to send gallbladder specimens for routine histopathological examination after cholecystectomy? The use of macroscopic examination. Dig Surg. 2013; 30 (4-6):472-5.

[26] Onzi TR1, d'Acampora AJ, de Araújo FM, Baratieri R, Kremer G, Lyra HF Jr, Leitão JT. Gastric histopathology in laparoscopic sleeve gastrectomy: pre- and post-operative comparison. Obes Surg. 2014 Mar; 24 (3):371-6. doi: 10.1007/s11695-013-1107-8.

[27] Renshaw AA, Rabaza JR, Gonzalez AM, et al. Helicobacter pylori infection in patients undergoing gastric bypass surgery for morbid obesity. Obes Surg. 2001; 11 (3):281-3. doi:10.1381/096089201321336601.

[28] Gnanapavan S, Kola B, Bustin SA, et al. The tissue distribution of the mRNA of ghrelin and subtypes of its receptor, GHS-R, in humans. J Clin Endocrinol Metab. 2002; $87: 2988$.

[29] Givon-Madhala O, Spector R, Wasserberg N, et al. Technical aspects of laparoscopic sleeve gastrectomy in 25 morbidly obese patients. Obes Surg. 2007; 17:722-7.

[30] Baltasar A, Serra C, Perez N, et al. Re-sleeve gastrectomy. Obes Surg. 2006; 16:1535-8.

[31] Goitein D, Goitein O, Feigin A, et al. Sleeve gastrectomy: radiologic patterns after surgery. Surg Endosc. 2009; 23:155963.

[32] Himpens J, Dobbeleir J, Peeters G. Long-term results of laparoscopic sleeve gastrectomy for obesity. Ann Surg. 2010; 252:319-24. 